\title{
Laboratory Testing for Substance Use Disorders
}

\author{
David Dadiomov
}

\section{High-Yield Review Points}

- Drug screening interpretation can be highly affected by patient-specific factors such as time since last use, metabolism, genetics, age, and weight.

- Knowledge of drug-specific factors such as half-life, tissue binding, and metabolism can help the clinician properly interpret a drug screening result.

- A thorough and accurate patient history is necessary prior to drug screening to interpret possible false-positives or negatives.

- Certain medications require baseline medical laboratory testing which should be conducted and followed to ensure safe use.

\section{Introduction}

Laboratory drug testing is an important tool utilized by clinicians for the comprehensive assessment of patients with substance use disorders. Drug screening gives a clinician objective evidence regarding the presence of various substances or metabolites with which inferences can be made about consumption of substances. Drug screens may be commonly utilized in nonmedical settings (such as for employment or legal purposes); however, it is important to discern these tests from those utilized in clinical practice. In the employment setting, false positives may carry severe implications, and thus detection limits for these are often higher. However, in the clinical setting, many factors may impact the result of a drug screen, and thus a greater level of detail is warranted in ordering and interpretation of laboratory results.

D. Dadiomov $(\triangle)$

University of Southern California School of Pharmacy, Los Angeles, CA, USA

e-mail: dadiomov@usc.edu 
Due in part to the societal stigma associated with drug screening, patients may have concerns that should be addressed. The reason for laboratory testing should be communicated, and both the patient and provider should have an agreement or understanding about how and why drug screens are conducted. In general, testing should be conducted in accordance with the patient's pattern of use, their stage in the recovery process, the substances used, and any practical considerations (such as transportation and schedule). This chapter will discuss the fundamentals of testing across different clinical scenarios.

Prior to interpreting urine drug screens, it is imperative that the clinician perform a thorough history of all products a patient has used and the time of last use. This medication history should include all licit, illicit, over-the-counter, and herbal products the patient may be using. Proper interviewing techniques (such as utilizing open-ended questions) should be employed to elicit a thorough history. Many substances have the potential to be detected as a false positive; these instances can be excluded if known. In addition, substances can vary significantly in their pharmacokinetic properties, and the timing of last use can help a clinician properly interpret results.

\section{Principles of Laboratory Testing}

Laboratory screening in SUD can be conducted for two major purposes, either to assess the safety of a given medication and conduct general medical testing or to assess the presence of substances or their metabolites. When conducting general medical testing, typically clinicians may be obtaining baseline labs to ensure a patient doesn't have a contraindication to a particular therapy (e.g., renal or hepatic impairment), as well as monitoring for laboratory abnormalities potentially caused by the medication. In contrast, when conducting drug screening the primary goal may be to assess that the patient is adherent to their prescribed regimen. It may also serve to assess whether a patient is diverting the medication or utilizing other substances. This chapter will focus primarily on drug screening; however, other elements of laboratory testing will be discussed when relevant.

A variety of drug screening tools are currently available to clinicians. Likely the most familiar of these tests will be immunoassay tests. These are usually "dipstick" urine tests that can be used for initial screening; however, they have lower sensitivity and specificity than confirmatory or laboratory tests via gas chromatography/mass spectroscopy (GCMS). Immunoassays are limited by their qualitative nature and higher proportion of false positive and false negative test results as compared to the quantitative tests. Urine immunoassays typically have a threshold, above which a specimen is considered to be positive. Typically, an immunoassay is used as a preliminary tool. If the test returns positive, it is usually sent for further confirmatory testing [1]. Despite several disadvantages, urine immunoassays are typically quick and easy to conduct and are inexpensive. Often immunoassays may be employed as a cost-savings tool when a high level of precision is not needed. 
Table 2.1 Federal urine drug screening cutoffs [1-3]

\begin{tabular}{l|l|l}
\hline Substance & Initial cutoff value & Typical urine detection window \\
\hline Marijuana (THC) & $50 \mathrm{ng} / \mathrm{mL}$ & $\begin{array}{l}\text { Single use }-2-3 \text { days } \\
\text { Moderate use }-5-7 \text { days } \\
\text { Chronic use }->30 \text { days }\end{array}$ \\
\hline Cocaine (benzoylecgonine) & $150 \mathrm{ng} / \mathrm{mL}$ & 3 days \\
\hline Codeine/morphine & $2000 \mathrm{ng} / \mathrm{mL}$ & 2 days \\
\hline Hydrocodone/hydromorphone & $300 \mathrm{ng} / \mathrm{mL}$ & 3 days \\
\hline Oxycodone/oxymorphone & $100 \mathrm{ng} / \mathrm{mL}$ & 3 days \\
\hline 6-Acetylmorphine & $10 \mathrm{ng} / \mathrm{mL}$ & 2 days \\
\hline Phencyclidine & $25 \mathrm{ng} / \mathrm{mL}$ & 7 days \\
\hline Amphetamine/methamphetamine & $500 \mathrm{ng} / \mathrm{mL}$ & 2 days \\
\hline MDMA/MDA & $500 \mathrm{ng} / \mathrm{mL}$ & 2 days \\
\hline
\end{tabular}

It is important for clinicians in addiction psychiatry to be aware of the detection limits of any test they are employing for clinical decision-making. The average detection limit for urine immunoassays tends to be relatively high to minimize the occurrence of false positives. Federal guidelines set guidelines for workplace urine drug screening cutoff levels (Table 2.1) [2]. In addition, the Department of Health and Human Services (HHS) has established a standard guideline for workplace drug screening, typically known as the "standard 5 panel drug test." This panel includes screening for marijuana, cocaine, phencyclidine (PCP), amphetamines, and certain opioids. As discussed further in this chapter, there are limitations to specific substances that can be detected on the standard drug screen.

Within addiction psychiatry, however, obtaining quantitative tests may be of greater clinical utility. With standard immunoassays, a patient's sample may be falsely negative for a variety of reasons including genotypic metabolism differences, a subthreshold positive result, or the particular substance or metabolite is not detected [1]. Furthermore, urine immunoassays may have many false positives and false negatives that limit their utility in addiction settings. A false-positive test may be the result of a substance used that has a similar chemical structure to the assayed chemical and thus shows up as a positive result. Agents that can cross-react for a false-positive test can be medications, herbal supplements, and food products.

Clinicians also have a variety of sampling methods for drug screenings (Table 2.2). Most commonly utilized are urine, blood, hair, nail, and breath tests. Urine tests are the most commonly employed due to their relative ease of collection, and good detection window for most substances or their metabolites. Blood sampling may be more useful for detecting acute intoxication as it has a shorter detection window. Conducting hair and nail testing leads to the longest detection window; however, there is also a delay for the substance to appear in the sample after use, and there are specific guidelines about how to collect hair and nail samples. Breath testing is also typically used primarily to detect acute intoxication of volatile substances such as ethanol and is very easy to administer in most environments [4].

In addition to ordering the best test for the setting and need, it is imperative that an addiction psychiatrist can interpret tests correctly. As mentioned previously, 
Table 2.2 Benefits and drawbacks of different drug screening methods

\begin{tabular}{l|l|l}
\hline $\begin{array}{l}\text { Sampling } \\
\text { method }\end{array}$ & Benefits & Drawbacks \\
\hline Urine & $\begin{array}{l}\text { Relatively inexpensive, readily available, } \\
\text { quick results, noninvasive sample } \\
\text { collection }\end{array}$ & $\begin{array}{l}\text { Limited accuracy for certain } \\
\text { compounds, adulteration risk if } \\
\text { unsupervised }\end{array}$ \\
\hline Blood & $\begin{array}{l}\text { May determine acute intoxication, } \\
\text { relatively inexpensive }\end{array}$ & $\begin{array}{l}\text { Invasive, will not determine past } \\
\text { drug use }\end{array}$ \\
\hline Hair & $\begin{array}{l}\text { Long period (3-6 months) of detecting } \\
\text { past use, noninvasive sample collection }\end{array}$ & $\begin{array}{l}\text { Will not detect recent drug use, } \\
\text { expensive }\end{array}$ \\
\hline Breath & $\begin{array}{l}\text { Long period of drug detection to hair test, } \\
\text { noninvasive collection }\end{array}$ & $\begin{array}{l}\text { Will not detect recent drug use, } \\
\text { expensive }\end{array}$ \\
\hline
\end{tabular}

Table 2.3 Potential positive results for substances of misuse [1]

\begin{tabular}{l|l}
\hline Substance & Potential positive result \\
\hline Amphetamines & $\begin{array}{l}\text { Aripiprazole, atomoxetine, bupropion, ephedrine, labetalol, MDMA, } \\
\text { phentermine, pseudoephedrine, ranitidine, selegiline, trazodone, Vicks } \\
\text { inhaler }\end{array}$ \\
\hline Benzodiazepines & Efavirenz, sertraline \\
\hline THC & Dronabinol, efavirenz, nabilone \\
\hline Opioids & Dextromethorphan, diphenhydramine, poppy seeds, quinolone antibiotics \\
\hline Phencyclidine & Dextromethorphan, doxylamine, ketamine, tramadol, venlafaxine \\
\hline
\end{tabular}

knowledge of full medication history is important as many substances may cause false positives in immunoassays. The clinician should consider the false-positive and false-negative rate of many available immunoassays (Table 2.3), though this can vary by manufacturer for each test. Knowledge of physical properties of various substances is important to being able to predict the results of a given drug screen. For instance, the metabolic pathway of a given substance may produce analytes that could incorrectly be interpreted as the use of a different substance if the interpreter doesn't recognize the full pathway. Information on specific substances will be discussed in this chapter. Other pharmacokinetic considerations should be noted, such as the half-life and tissue binding of a substance and/or its metabolites which would dictate the time frame that a test may be valid. Finally, individual pharmacogenomics may alter the analytes in a specimen as well. This may occur when individuals are poor or ultrarapid metabolizers of a substance.

Providers should also consider ways of manipulation of the sample if there is an incentive to do so. While some samples are less likely to demonstrate interference (such as blood or breath tests), unobserved urine samples may lend themselves to adulteration. In these instances, a urinalysis is recommended to provide information for interpretation. The sample would be expected to have a temperature close to normal body temperature. The color of the sample, urine creatinine, specific gravity, and $\mathrm{pH}$ should be within normal homeostatic ranges (Table 2.4) [5]. 
Table 2.4 Homeostatic urinalysis ranges

\begin{tabular}{l|l}
\hline Criteria & Range \\
\hline Urine creatinine & $18-200 \mathrm{mg} / \mathrm{dL}$ \\
\hline $\mathrm{pH}$ & $4.5-8.0$ \\
\hline Specific gravity & $1.002-1.030$ \\
\hline Temperature & $90-100^{\circ} \mathrm{F}$ \\
\hline
\end{tabular}

\section{Drug-Specific Information}

\section{Opioids}

Opioids broadly describe the substances that have agonist activity at the mu opioid receptors, as well as other opioid receptors. Naturally occurring opioids can be further classified as opiates, whereas semisynthetic and fully synthetic opioids require some manipulation for their manufacture and are chemically distinct. Opioids contain differing pharmacokinetic properties which can vary depending on their specific formulation (e.g., extended release tablets). These different properties carry implications for detection in drug screens. For instance, opioids that are formulated in a long-acting or extended release formulation may be detectable in specimens for a longer period. Additionally, most urine drug screens are not as sensitive for semisynthetic opioids such as oxycodone and hydrocodone nor synthetic opioids such as fentanyl. In instances where the clinician wishes to test for those opioids, a specific test will need to be ordered.

Another important consideration for those caring for patients with opioid use disorders is the metabolism of the different opioids. Figure 2.1 demonstrates a general pathway for opioid metabolism. Of note, the opioid metabolism pathway of many opioids may converge at several common metabolites. For instance, a patient taking either codeine, morphine, or heroin may produce a urine specimen that contains morphine. Therefore, a patient that is prescribed acetaminophen and codeine tablets, and takes a urine drug screen (UDS) that shows a positive result for morphine, should not necessarily be accused of use of other substances. Similarly, utilizing various analytes and their concentrations may help discern use of one substance from the other. A patient that is abusing heroin (aka diacetyl morphine) would show morphine in their UDS, just as a patient taking morphine or codeine would. However, a test for the analyte 6-monoacetyl morphine is specific to the heroin metabolite and would indicate heroin use. However, 6-monoacetyl morphine has a fairly short detection window in the urine of 8 hours [1].

Providers should be aware that many synthetic opioids (such as fentanyl, methadone, or buprenorphine) do not show up on standard drug panel and will typically require a specific lab test for these agents [1]. If taking care of patients who may be using synthetic opioids, it is important to ensure that the capability to order these specific lab tests exists. 
Fig. 2.1 Opioid metabolism [6]

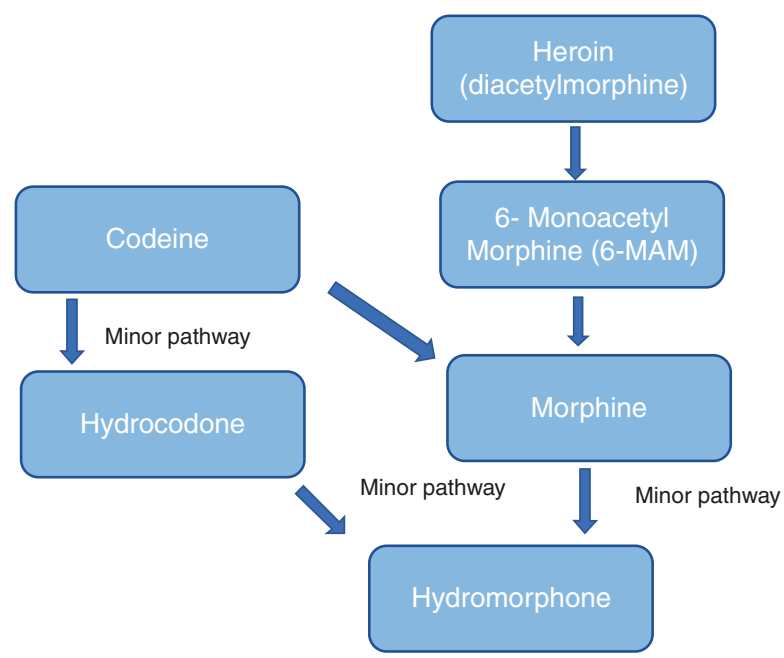

Buprenorphine is a synthetic opioid commonly used in both treatment of pain as well as treatment of opioid use disorder. Clinicians may wish to order buprenorphine urine testing for several reasons. Since it is a mu-opioid partial agonist, there is a potential for buprenorphine misuse. Patients may be acquiring illicit buprenorphine and the provider wishes to assess the use pattern. Another reason for buprenorphine drug screening is to ensure adherence to the regimen for opioid use disorder. This serves as a marker of medication ingestion and to minimize diversion of buprenorphine. Urine testing of buprenorphine/naloxone products should include buprenorphine and norbuprenorphine (major metabolite) [7]. Providers should carefully consider the pattern of detection that would be expected with adherence as compared to adulterated samples. A patient taking buprenorphine/naloxone formulation would be expected to produce a sample containing buprenorphine and norbuprenorphine. Additionally, some laboratories may report naloxone concentrations; a proportion of the naloxone may be absorbed sublingually and thus be detectable in the urine. Some laboratories may report naloxone concentrations as either free naloxone, naloxone-3-glucuronide, or total naloxone concentrations $[8,9]$. Analysis of free naloxone in urine may indicate adulteration, whereas naloxone-3-glucuronide is the expected analyte from first pass metabolism of oral or sublingual naloxone.

Methadone is another synthetic opioid that is commonly used for both treatment of pain as well as opioid use disorder. Again, special laboratory testing is required to detect methadone in the urine. Methadone testing is prone to adulteration of urine with drug. To determine if methadone was added to urine (as opposed to being present via excretion of the drug into urine), clinicians may choose to order a test for methadone's metabolite 2-ehtylidene-1,5-dimethyl-3,3-diphenylpyrollidine (EDDP). The presence of EDDP would indicate that the patient has ingested and metabolized methadone [1]. 


\section{Alcohol}

Alcohol is one of the most commonly used substances and may often co-occur with other substance use or mental health disorders. Alcohol has slightly different pharmacokinetics from many other substances. In general, ethanol undergoes metabolism that is termed "zero-order" kinetics, or saturable pharmacokinetics. This implies that the metabolism of alcohol occurs at a given metabolic rate $(20 \mathrm{mg} / \mathrm{dL} / \mathrm{hour})$ regardless of the concentration of alcohol in the body; thus, the metabolism is "saturable." Several factors may affect this metabolic rate such as sex (women eliminate alcohol faster, though they have higher blood alcohol concentrations because of a smaller volume of distribution), race, whether someone is in the fed nutritional state, and body mass [10].

The usual laboratory measurement for alcohol in clinical settings is the blood alcohol content (BAC) expressed as mg of ethanol per $\mathrm{dL}$ of blood. BAC may be recognized as its expression of a percentage, as in the legal limit to operate a motor vehicle that is $0.08 \%$. A BAC of $0.1 \%$ corresponds to $100 \mathrm{mg}$ ethanol/dL blood. A "standard drink" in the United States is defined as $14 \mathrm{~g}$ of ethanol, or 0.6 fluid ounces of pure ethanol. Similarly, this corresponds to a 12 oz beer ( $5 \% \mathrm{ABV})$, a $5 \mathrm{oz}$ glass of wine (12\% ABV), or $1.5 \mathrm{oz}$ of spirit (40\% ABV).

BAC is easily estimated using a breathalyzer machine which can give clinicians a quick reading of estimated BAC. Due to the ease and affordability of testing, the comorbid presence of alcohol use disorders with other substance use disorders, and the negative implications of alcohol intoxication to recovery, many clinicians will utilize a breathalyzer in routine outpatient follow-up. In addition, patients newly presented to inpatient units or emergency departments may obtain a blood draw to determine their alcohol content to guide further treatment considerations.

In addition to labs to detect acute intoxication, several other forms of laboratory testing may be utilized to assess patients with alcohol use disorders. While ethanol has a fairly short half-life, it is metabolized to ethyl glucuronide and ethyl sulfate which are excreted in the urine. Ethyl glucuronide may be detected for up to 3-4 days after alcohol consumption. Ethyl sulfate may be used concurrently to increase the specificity of the ethyl glucuronide test [11]. Another test for ethanol consumption utilized is phosphatidyl ethanol. Phosphatidyl ethanol is elevated in the presence of 4-5 standard drinks per day for at least 3 weeks. It remains elevated for up to 14 days from discontinuing alcohol [11]. Gamma glutamyl transferase (GGT) is a liver enzyme that is increased in individuals with chronic heavy alcohol use. In patients without chronic heavy alcohol use, GGT levels are expected to be below 54 IU/L. It should be noted that certain anticonvulsant medications may increase a patients GGT even without alcohol use. GGT may also be increased in certain hepatic conditions, and so it is not specific for alcohol use. Carbohydratedeficient transferrin (CDT) is a protein that exists in blood and is increased with heavy alcohol use. Elevations in carbohydrate-deficient transferrin may be noted sooner than elevations in GGT. A value greater than $1.6 \%$ indicates heavy alcohol consumption. Finally, standard liver function panel labs may be assessed in alcohol use disorder. Liver function tests (LFTs) such as aspartate aminotransferase (AST) and alanine aminotransferase (ALT) and mean corpuscular volume (MCV) may be increased with repeated alcohol use. Elevations in LFTs have been associated with 
hepatic cellular damage due to toxins, and MCV elevations are more likely a result of direct hematologic toxicity of ethanol, rather than vitamin deficiencies. These tests are not as sensitive or specific as those previously listed [11].

\section{Benzodiazepines}

Benzodiazepines are also commonly used agents for anxiety and related disorders that have also been widely misused. As with other substances, it is important to consider the pharmacokinetics of the specific agent that is being detected. Benzodiazepines vary in their half-lives with some having a half-life as short as 10 hours (alprazolam), and others lasting 3 days or longer (diazepam). Diazepam has a half-life of 72 hours; however, its active metabolite desmethyldiazepam has a half-life of 160 hours. Due to the drug lingering in the body for so long, diazepam may be detected for up to 30 days [1].

Urine immunoassays for benzodiazepines unfortunately have a high rate of false negatives. Most assays are designed to detect diazepam or its metabolites such as nordiazepam, or oxazepam. Benzodiazepine metabolites as a result of glucuronidation in liver metabolism (such as metabolites of alprazolam or lorazepam) are often not detected on standard immunoassays. Similarly, clonazepam is metabolized by nitroreducation, and its metabolite is not often detected (Fig. 2.2). A small proportion

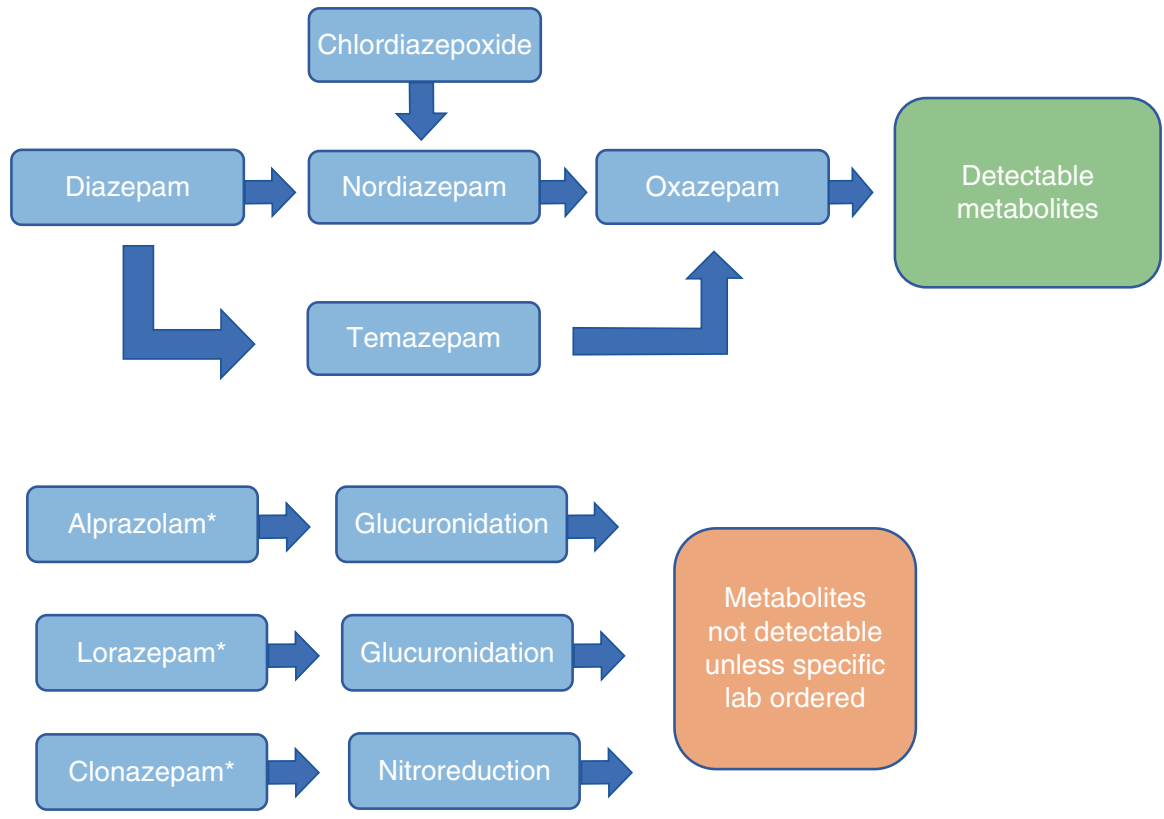

${ }^{*}$ A small minority of these benzodiazepines are excreted unchanged in the urine. Even without specific lab testing, they may be detected but usually with less sensitivity and at high concentrations.

Fig. 2.2 Benzodiazepine metabolites 
of these benzodiazepines (alprazolam, lorazepam, and clonazepam) are excreted in the urine unchanged and may therefore be detected by urine drug screens. Careful consideration is warranted for the assays cutoff value for detection. Potent agents requiring lower doses (such as alprazolam or lorazepam) may not show up on an assay if the cutoff for detection is $300 \mathrm{ng} / \mathrm{mL}$. Agents that undergo metabolism that is not readily detectable on immunoassay further decrease the likelihood of detection as only a small proportion of the original dose is available to be detected.

Tests that are specific for these compounds can be helpful in detecting an individual's use of benzodiazepines. In some instances, lower detection cutoffs may be available and useful for detecting potent or glucuronidated benzodiazepines. Finally, some laboratories are able to enzymatically alter the benzodiazepine metabolites to the parent drug via hydrolysis of the urine sample [1].

\section{Marijuana}

Urine drug screens focus on detecting a metabolite of the main psychoactive component of marijuana, delta-9 tetrahydrocannabinol (THC). Typically, the federal cutoff limit for detection is $50 \mathrm{ng} / \mathrm{mL}$ [2]. Several factors may affect how long THC may be detectable in an individual. The first consideration should be whether the user is a chronic smoker or not. In infrequent users, THC may be detectable for only 3 days. However, chronic heavy users may be detectable for 30 days or longer due to elution of THC that was stored in body tissues. A positive immunoassay will not be able to distinguish acute intoxication from use that may have occurred several days ago.

A commonly held misconception about marijuana is that passive inhalation (such as from a concert) may produce a positive result. This has been tested in a controlled environment, and while nonsmokers in an enclosed environment had some THC metabolites detectable on GCMS (mean ranging from 7.5 to $28.3 \mathrm{ng} / \mathrm{mL}$ depending on THC concentration in marijuana and whether ventilation was in the room). The results demonstrated that only 1 of the 6 subjects had a positive result above the federal threshold of $50 \mathrm{ng} / \mathrm{mL}$ with high-potency marijuana in an enclosed environment with no ventilation. The result was only positive at 4-6 hours post exposure, and subsequent specimens fell below $50 \mathrm{ng} / \mathrm{mL}$. Therefore, it is highly unlikely to test positive for passive inhalation in an instance where exposure is not obvious [12].

\section{Synthetic Cannabinoids}

Synthetic cannabinoids have been developed and widely used due to their similar effects to cannabinoids and their difficulty in detection on standard drug tests. Synthetic cannabinoids represent a diverse array of chemicals that are often sprayed onto inert plant material and smoked in a similar fashion to marijuana [13]. The DEA has banned many of these synthetic compounds because of numerous poison center reports [1]. Despite the fact that standard federal panels do not test for 
synthetic cannabinoids, there is increasing development of urine immunoassays to detect these substances. Several validated immunoassays are available, but it is important for clinicians to recognize that not all synthetic cannabinoids have an associated screening tool and further development of novel cannabinoids may occur in the future. The usual detection time reported for synthetic cannabinoids is 72 hours; however, this detection window may be increased in chronic users [1].

\section{Stimulants}

Stimulant medications are commonly prescribed for disorders such as ADHD, though they are frequently misused as well. This class includes drugs such as amphetamine, methylphenidate, methamphetamine, and cocaine. UDS for stimulants can be challenging for clinicians due to the poor selectivity of these assays, specifically those screening for amphetamines. Due to chemical similarities, the distinction between amphetamine and methamphetamine is difficult to make without a more quantitative laboratory analysis [1]. Clinicians should be aware that many prescribed and over-the-counter medications may cause false positives (and even some "true" positives) on UDS. For instance, the MAOI selegiline is metabolized to methamphetamine in the body and may trigger a positive UDS. Oxymetazoline (Vicks) nasal spray has also been shown to produce a positive urine screen after following manufacturer-recommended dosing [1]. False positives for amphetamine screens may include aripiprazole, atomoxetine, bupropion, chlorpromazine, ephedrine, metformin, MDMA, phentermine, pseudoephedrine, ranitidine, and trazodone, among others. The standard federal 5 panel drug screen includes confirmatory testing for MDMA (methalenedioxymethamphetamine or "ecstasy") as well as MDA (methylenedioxyamphetamine). If a urine screen is positive for amphetamines, it is recommended to utilize quantitative confirmatory testing (e.g., GCMS testing) to verify the result. Amphetamines or methamphetamines are typically detected in the urine for about 48-72 hours after use [1,3]. It should be noted that to detect methylphenidate accurately, a specific laboratory test for it should be ordered.

Cocaine, on the other hand, is a urine screen that has a high level of specificity, and no false positives have been identified for this assay [1,3]. Cocaine assays screen for benzoylecgonine: a metabolite of cocaine. It is detectable in the urine for 2-4 days after use [1]. Cocaine is a schedule II substance by the FDA and is often used licitly as an eye drop in ophthalmic procedures. It is reasonable that use under these circumstances may produce a positive test result; however, other aminoester or aminoamide local anesthetics (such as benzocaine and lidocaine) do not produce false-positive results [1].

Nicotine is the legal stimulant present in tobacco leaves and also sold over-thecounter in gums, patches, and lozenges. It is present in many "vapes" as a heated and inhaled liquid. Many patients who misuse substances are concomitantly dependent on nicotine. When assessed, providers are able to order a urine screen on nicotine's major metabolite, cotinine. Cotinine can be detected in the urine of heavy smokers for up to two weeks [14]. 


\section{Dissociatives}

Dissociative drugs include phencyclidine (PCP), ketamine, and dextromethorphan. These agents produce perceptual disturbances, hallucinations, and characteristic out of body (dissociative) experiences. PCP is included in the standard federal 5 panel drug test. While PCP is a schedule I controlled substance, ketamine and dextromethorphan are commonly used in medical care. These agents have the potential to show up as a false positive for PCP. The use of these agents should be excluded prior to interpreting a positive UDS. PCP is typically detected in the urine for up to a week following its use [3].

\section{Hallucinogens and Other Drugs}

Hallucinogens include substances such as psilocybin (active ingredient in psychedelic mushrooms), lysergic acid diethylamide (LSD), and mescaline (found in peyote cactus) have not historically been detected on standard urine drug screens. In many instances, these substances have short half-lives or are sufficiently potent that only a small amount of drug is needed for recreational use. While specialized laboratory testing is available, it is fairly uncommon in clinical practice. Other substances besides hallucinogens that may be difficult to detect on a urine screen include gamma-hydroxybutyrate (GHB), inhalants, and synthetic cathinones ("bath salts") [3].

\section{Review Questions}

1. A patient enrolled in your opioid use disorder clinic presents for a routine followup and refill for their buprenorphine prescription. The patient submits a urine drug screen prior to the appointment. The clinician reviews the results which indicate a positive result for morphine and 6-monoacetyl morphine. What is the most likely substance this patient was using?
A. Morphine
B. Heroin
C. Codeine
D. Oxycodone
E. Hydromorphone

\section{Correct answer: B. Heroin}

Explanation: Heroin, or diacetyl morphine, is converted in the body to 6-monoacetyl morphine and then to morphine. Morphine use alone would not explain the presence of 6-monoacetyl morphine. Codeine would show up as codeine and morphine. Oxycodone and hydromorphone are semisynthetic opioids that would not be expected to contain morphine nor 6-monoacetyl morphine in their metabolic pathway. 
2. A patient presents to the emergency department for aberrant behavior. The clinical staff suspect the patient's behaviors may be due to ingestion of substances. The physician on the team orders a urine drug screen, but would like to consider substances that may not be readily detectable by urine immunoassay. Identify the substance that is least likely to be detected on a standard urine drug screen.
A. Phencyclidine
B. Marijuana
C. Diazepam
D. Bath salts
E. Mixed amphetamine salts

Correct answer: D. Bath salts

Explanation: Bath salts are not one of the standard items that can be detected on urine drug screens, and would require highly specialized tests to detect. PCP, marijuana, diazepam, and amphetamines are all detected as part of the standard federal 5 panel drug test.

3. A patient is seen for detoxification of alcohol; however, the patient is not able to give a reliable medical or social history. The medical team would like to determine whether the extent to which the patient has been consuming alcohol. Which laboratory test would best identify a patient that has been using alcohol excessively over a prolonged period?
A. Phosphatidylethanol
B. Ethyl glucuronide
C. Alcohol breath test
D. Albumin
E. Serum creatinine

Correct answer: A. Phosphatidylethanol

Explanation: Phosphatidylethanol, elevated GGT, or carbohydrate-deficient transferrin can be used to identify patients with prolonged alcohol use. Ethyl glucuronide is useful for detecting use within past 3-4 days, and alcohol breath test for patients that have recently ingested alcohol. The albumin and serum creatinine are not used to assess alcohol use.

4. A 31-year-old male presents to the addiction clinic for treatment of opioid use disorder. The patient claims to use opioids regularly and would like to "get clean." He submits a urine sample in the office for a standard immunoassay. The results come back and are negative for opiates, benzodiazepines, PCP, marijuana, and amphetamines. The physician runs the state prescription drug monitoring program and discovers the patient has had multiple pharmacy refills in the past month for fentanyl and alprazolam. Which of the following statements is correct regarding the results of this patient's immunoassay?

A. The patient is likely not using any substances and is seeking buprenorphine for diversion.

B. The cutoff values on the immunoassay are likely too low to detect any use of fentanyl and alprazolam. 
C. Fentanyl and alprazolam may not be detected on a standard urine immunoassay.

D. The immunoassay would only detect chronic use of these substances.

E. The immunoassay should be repeated to ensure accuracy of results.

Correct answer: C. Fentanyl and alprazolam may not be expected to be positive on a standard urine immunoassay.

Explanation: Synthetic opioids (such as fentanyl) and certain benzodiazepines (lorazepam, alprazolam, clonazepam) are unlikely to be detected on standard immunoassays that were developed for morphine and diazepam. While diversion may be a concern, we can't rule out this patient is not taking these medications. A substance-specific test via GCMS should be performed. For alprazolam, the clinician can consider lowering the limit of detection in the laboratory or adding an enzyme to the urine sample to convert the undetectable metabolite to the parent drug as well.

5. A 41-year-old female presents as a follow up to the clinic. Her routine urine drug screen immunoassay shows a positive result for amphetamine. The patient states that she has been ill with a sinus infection and has been taking amoxicillin, pseudoephedrine/cetirizine combination, and dextromethorphan. The patient is adamant that she did not use any amphetamine or methamphetamine products. What is the most appropriate action for the clinician regarding the positive UDS?

A. Discontinue the patient from the treatment program for violating the substance use agreement

B. Ignore the results since the patient is taking several medications which can cause a false positive

C. Order confirmatory GCMS testing since dextromethorphan can cause a false positive for amphetamines

D. Order confirmatory GCMS testing since Amoxicillin can cause false positive for amphetamines

E. Order confirmatory GCMS testing since pseudoephedrine can cause false positive for amphetamines

Correct answer: E. Order confirmatory GCMS testing since pseudoephedrine can cause false positive for amphetamines

Explanation: Amphetamine immunoassays can have many false positives, and pseudoephedrine is one of them. Amoxicillin and dextromethorphan do not cause false positives for amphetamines. While there is a plausible cause for the positive result, confirmatory testing should be conducted to ensure this wasn't a true positive result.

\section{References}

1. Moeller KE, Kissack JC, Atayee RS, Lee KC. Clinical interpretation of urine drug tests: what clinicians need to know about urine drug screens. Mayo Clin Proc. 2017;92(5):774-96.

2. US Department of Health and Human Services, Substance Abuse and Mental Health Services Administration. Mandatory guidelines for federal workplace drug testing programs: notices. Federal Register. 82. 2017;13:7920-70. 
3. Nelson ZJ, Stellpflug SJ, Engebretsen KM. What can a urine drug screening immunoassay really tell us? J Pharm Pract. 2016;29(5):516-26.

4. Verstraete AG. Detection times of drugs of abuse in blood, urine, and oral fluid. Ther Drug Monit. 2004;26(2):200-5.

5. Cook JD, Caplan YH, Lodico CP, Bush DM. The characterization of human urine for specimen validity determination in workplace drug testing: a review. J Anal Toxicol. 2000;24(7):579-88.

6. Pesce A, West C, Egan city K, Strickland J. Interpretation of urine drug testing in pain patients. Pain Med. 2012;13(7):868-85.

7. Hull MJ, Bierer MF, Griggs DA, Long WH, Nixon AL, Flood JG. Urinary buprenorphine concentrations in patients treated with suboxone as determined by liquid chromatography-mass spectrometry and CEDIA immunoassay. J Anal Toxicol. 2008;32(7):516-21.

8. Danso D, Langman LJ, Jannetto PJ. Targeted opioid screening assay for pain management using high-resolution mass spectrometry. Methods Mol Biol. 1872;2019:41-50.

9. Fang WB, Chang Y, Mccance-katz EF, Moody DE. Determination of naloxone and nornaloxone (noroxymorphone) by high-performance liquid chromatography-electrospray ionizationtandem mass spectrometry. J Anal Toxicol. 2009;33(8):409-17.

10. Jones AW. Pharmacokinetics of ethanol - issues of forensic importance. Forensic Sci Rev. 2011;23(2):91-136.

11. Tavakoli HR, Hull M, Michael Okasinski L. Review of current clinical biomarkers for the detection of alcohol dependence. Innov Clin Neurosci. 2011;8(3):26-33.

12. Cone EJ, Bigelow GE, Herrmann ES, et al. Non-smoker exposure to secondhand cannabis smoke. I. Urine screening and confirmation results. J Anal Toxicol. 2015;39(1):1-12.

13. Arntson A, Ofsa B, Lancaster D, Simon JR, Mcmullin M, Logan B. Validation of a novel immunoassay for the detection of synthetic cannabinoids and metabolites in urine specimens. J Anal Toxicol. 2013;37(5):284-90.

14. Vine MF, Hulka BS, Margolin BH, et al. Cotinine concentrations in semen, urine, and blood of smokers and nonsmokers. Am J Public Health. 1993;83(9):1335-8.

Open Access This chapter is licensed under the terms of the Creative Commons Attribution 4.0 International License (http://creativecommons.org/licenses/by/4.0/), which permits use, sharing, adaptation, distribution and reproduction in any medium or format, as long as you give appropriate credit to the original author(s) and the source, provide a link to the Creative Commons license and indicate if changes were made.

The images or other third party material in this chapter are included in the chapter's Creative Commons license, unless indicated otherwise in a credit line to the material. If material is not included in the chapter's Creative Commons license and your intended use is not permitted by statutory regulation or exceeds the permitted use, you will need to obtain permission directly from the copyright holder.

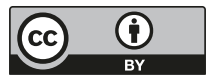

